\title{
Frontières
}

\section{Le droit des vivants de choisir lorsque leur mort s'annonce}

\section{Lina B. Moreco}

Volume 14, numéro 2, printemps 2002

La mort prononcée

URI : https://id.erudit.org/iderudit/1073978ar

DOI : https://doi.org/10.7202/1073978ar

Aller au sommaire du numéro

Éditeur(s)

Université du Québec à Montréal

ISSN

1180-3479 (imprimé)

1916-0976 (numérique)

Découvrir la revue

Citer ce document

Moreco, L. B. (2002). Le droit des vivants de choisir lorsque leur mort s'annonce. Frontières, 14(2), 82-83. https://doi.org/10.7202/1073978ar d'utilisation que vous pouvez consulter en ligne.

https://apropos.erudit.org/fr/usagers/politique-dutilisation/ 


\section{LE DROIT DES VIVANTS DE CHOISIR LORSQUE LEUR MORT S'ANNONCE}

\author{
Lina B. Moreco, \\ réalisatrice de documentaires.
}

\section{MOURIR POUR SOI}

En février 2001 sortait en salle mon film documentaire: Mourir pour soi ${ }^{1}$. Dans ce film, j'ai essayé de comprendre ce qu'est notre liberté d'agir concernant la fin de notre vie (acharnement thérapeutique, arrêt de traitement, refus du maintien artificiel de la vie et aide à mourir / euthanasie), tout en proposant une réflexion sur le sens des mots humanité, dignité et liberté.

Et puis le film a choqué. Il a irrité certains membres du milieu médical, ceux qui précisément détiennent le pouvoir sur notre santé et sur notre mort. Ce qui importe, selon plusieurs médecins, «c'est le respect de la vie non pas la liberté de mourir; en développant les soins palliatifs, toute souffrance, qu'elle soit physique, psychologique ou spirituelle pourra être soulagée et plus personne ne demandera à être aidé à mourir ». On sous-entend aussi qu'intervenir dans le processus de fin de vie reviendrait à voler la mort d'une personne et à la détourner d'une expérience personnelle enrichissante.

\section{MOURIR AUJOURD'HUI}

Pourtant, mourir aujourd'hui, c'est bien souvent mourir inconscient, intubé, gavé, perfusé, anesthésié, ce qui est bien loin du tableau de la belle mort proposée par les soins palliatifs. Même si vous êtes âgé de 95 ans, atteint de la maladie d'Alzheimer, avec une perte d'autonomie totale, vous serez branché à une machine de gavage, vous recevrez de fortes médications, des traitements extraordinaires, parfois douloureux, afin de vous maintenir en vie, de ne pas vous laisser mourir. Une situation où l'on a peine à regarder la mort en face, à l'envisager comme la fin inéluctable qu'elle n'a jamais cessée d'être, où l'on préfère le prolongement de la mort et non pas la qualité de la vie.

Donc, bien avant que nous soient offerts les soins palliatifs (qui ont pour but de nous aider à bien mourir sans prolonger indûment la vie), nous faisons face à une médecine qui nous conditionne à outrepasser ce que nous pressentons du déclin naturel de la vie en nous proposant des solutions de survie à tout prix. Un contexte d'immortalité du corps où peu à peu nous sommes dépossédés de notre existence, manipulés jusqu'au plus profond de notre intimité, infantilisés par tous ceux qui travaillent pour nous et, finalement, emprisonnés dans le silence de notre corps. Une fin de vie où nous n'avons plus de responsabilités si ce n'est celle de ne pas trop déranger par notre perte de dignité et par la douleur de nos souffrances.

En fait, le drame actuel, c'est notre état de dépendance à l'égard de la médecine officielle. Déresponsabilisé de notre santé depuis longtemps, nous agissons en consommateurs non avertis qui cherchent à guérir plutôt qu'à prévenir les maladies. Conséquemment, nous sommes à la merci de ceux qui ont choisi la mort pour métier, puisque nous avons préféré ignorer que la mort est notre lot à tous. Nous laissons la porte grande ouverte aux abus et aux erreurs et nous encourageons le dépérissement de notre système de santé au profit d'une médecine de l'argent.

\section{LA VIE, UN ENJEU ÉCONOMIQUE}

Notre système de santé coûte de plus en plus cher, les hôpitaux sont constamment engorgés et le nombre de personnes malades augmente. Pourtant, les entreprises de produits pharmaceutiques ne cessent d'investir dans la recherche médicale, la découverte de médicaments miracles et l'application de nouveaux protocoles. Leurs profits dépassent maintenant ceux des banques. Simultanément, les hôpitaux et le cabinet des médecins sont devenus un réseau de recrutement et de distribution des plus efficaces pour vendre les produits, expérimenter les traitements, recommander des chirurgies et rentabiliser les investissements.

C'est dans ce contexte lucratif que les grands malades, les enfants et les personnes âgées deviennent des proies faciles, des cobayes... conformément au mot d'ordre: "la vie à tout prix ». On veut leur sauver la vie. Il ne faut donc pas se surprendre que l'on veuille aussi nous faire croire que le patient qui dit «non » aux traitements et qui demande qu'on le laisse mourir paisiblement soit atteint d'une pathologie qui se rapproche de la maladie mentale ou de la dépression. On déclare alors qu'il est nécessairement incapable de décisions claires et que vouloir mourir est aussi une maladie qui se soigne.

La science nous ayant pris en otage, nous sommes devenus ses objets forcés.

C'est en transformant notre rapport à la mort que nous pourrons y faire face sainement. Il ne faut plus la laisser au hasard; il ne faut plus s'en remettre aveuglément aux médecins ou à la famille. Ne plus avoir peur de la mort, qu'elle ne soit plus vue comme un échec que l'on cherche à cacher. Savoir que si nous ne pouvons disposer de cette vie qui nous quitte, c'est parce qu'elle ne nous appartient plus et que si nous voulons mieux mourir, il faudra reconquérir la mort.

\section{LE MOURIR LIBRE, UNE RÉALITÉ RÉCONFORTANTE}

Nous laisser libre de mourir en temps opportun (Pohier, 1998), c'est nous laisser libre de mourir en accord avec ce que nous sommes. Un médecin généraliste français, qui a aidé quelques personnes à mourir selon leur volonté témoigne que «L'euthanasie augmente la qualité de vie de la personne malade et que cela peut aussi augmenter la durée de la vie» (Leguay, 2000). Selon ce médecin, la personne qui est 
écoutée et respectée dans sa volonté de mourir dignement en arrive à mieux vivre sa maladie, car elle se sait en sécurité. Cela donne à la souffrance, qui n'a pas tellement de sens, un tout autre visage. Cette porte ouverte sur notre mort, sur la liberté d'aller la rejoindre, rassure; l'angoisse de souffrir encore et davantage, sans savoir jusqu'à quand, disparaît, transformant le sens même de l'expérience de la maladie. Ainsi apaisée de sa peur de souffrir, il se peut que la personne dépasse le temps de vie prévu par les médecins.
Il faut nous réapproprier notre mort; ne plus la laisser à l'extérieur de nous, ni contraire à nous, qu'elle ne soit plus un adversaire contre lequel il faut lutter, mais au contraire, une étape normale de notre vie, que nous réussirons à intérioriser, à mieux vivre et à partager avec nos proches.

\section{ÉCRIRE NOS VOLONTÉS}

Lorsque Nancy B., en 1992, obtint de la Cour d'appel, l'autorisation que soit débranché le respirateur qui la maintenait artificiellement en vie, ce qui a entraîné sa

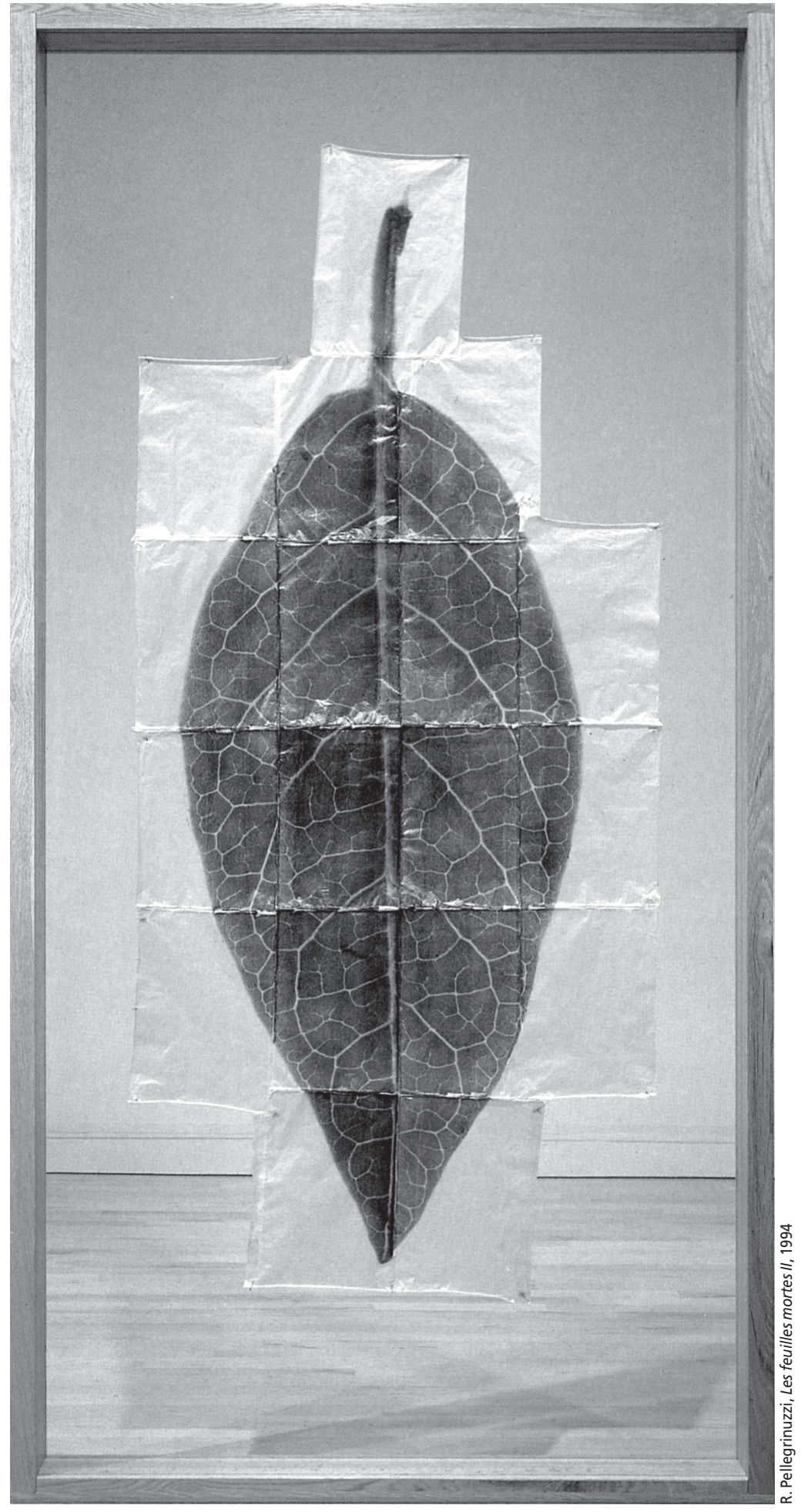

mort, le Code civil du Québec ${ }^{2}$, article 10, concernant le droit des malades, fut réinterprété. Depuis le procès, on nous reconnaît le droit de refuser tout maintien artificiel de la vie, en pourvu que nous soyons capables de le dire.

Il existe deux outils qui peuvent nous aider à exprimer nos volontés. Le mandat en cas d'inaptitude et le testament biologique $^{3}$. Écrire un testament biologique, c'est ouvrir le dialogue avec nos proches, faire connaître nos volontés de fin de vie et nous protéger de tout acharnement familial ou médical (test, médicamentation, expérimentation, chirurgie et maintien artificiel de la vie). Quant au mandat en cas d'inaptitude, il permet de désigner les personnes qui prendront des décisions à notre place, si nous ne sommes plus capables de le faire.

Exprimer nos volontés par écrit est un geste de communication des plus significatifs et des plus dynamiques que nous pouvons exécuter de notre vivant. C'est s'accorder une garantie de liberté et se donner le droit de mourir dignement; c'est demander que sa volonté et son libre choix soient respectés.

Chaque personne, lorsqu'elle est confrontée à la maladie et à la mort, doit passer à travers ses peurs, faire le deuil d'une vie passée pour finalement accepter la mort comme un accomplissement final. Parler de liberté de mourir, c'est reconnaître qu'il y a différentes façons de vivre tout comme il y a différentes manières de mourir; deux mouvements qui exigent notre plus grand respect. Il nous faut accepter que sur cette route il $\mathrm{y}$ ait des malades qui souffrent, à un stade de la maladie où d'autres n'ont rien manifesté. C'est dans le respect du malade, dans l'écoute de sa différence qu'il nous sera possible d'offrir l'accompagnement dont chacun a besoin.

\section{Bibliographie}

LEGUAY, Catherine (2000). Mourir dans la dignité. Quand un médecin dit oui, Paris, Robert Laffont.

POHIER, Jacques (1998). La mort opportune. Les droits des vivants sur la fin de leur vie, Paris, Seuil.

\section{Notes}

1. Mourir pour soi. Office national du film du Canada. http://www.onf.ca/mourirpoursoi

2. Code civil du Québec, chapitre 10.

3. Un mandat en cas d'inaptitude, signé chez le notaire, est aujourd'hui un document légalement reconnu. Par contre, le testament biologique n'a pas encore de valeur légale; il est difficilement reconnu par certains médecins, mais il faut savoir que la tendance actuelle est de reconnaître sa valeur. 